\title{
Simultaneous Image Color Correction and Enhancement using Particle Swarm Optimization
}

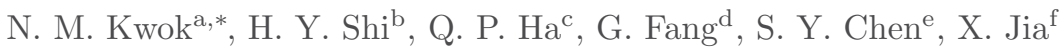 \\ ${ }^{a}$ School of Mechanical and Manufacturing Engineering \\ The University of New South Wales, Australia \\ ${ }^{b}$ School of Computer Science and Technology \\ Shaoxing University, Shaoxing, China \\ ${ }^{c}$ School of Electrical, Mechanical and Mechatronic Systems \\ University of Technology Sydney, Australia \\ ${ }^{d}$ School of Engineering \\ University of Western Sydney, Australia \\ ${ }^{e}$ College of Computer Science \\ Zhejiang University of Science and Technology, China \\ ${ }^{f}$ School of Engineering and Information Technology, University College \\ The University of New South Wales, Australia
}

\begin{abstract}
Color images captured under various environments are often not ready to deliver the desired quality due to adverse effects caused by uncontrollable illumination settings. In particular, when the illuminate color is not known a priori, the colors of the objects may not be faithfully reproduced and thus impose difficulties in subsequent image processing operations. Color correction thus becomes a very important pre-processing procedure where the goal is to produce an image as if it is captured under uniform chromatic illumination. On the other hand, conventional color correction algorithms using linear gain adjustments focus only in color manipulations and may not convey the maximum information contained in the image. This challenge can be posed as a multi-objective optimization problem that simultaneously corrects the undesirable effect of illumination color cast while recovering the information conveyed from the scene. A variation of the particle swarm optimization algorithm is further developed in the multi-objective optimization perspective that results in a solution achieving a desirable color balance and an adequate delivery of information. Experiments are conducted using a collection of color images of natural objects that were captured under different lighting conditions. Results have shown that the proposed method is capable of delivering images with higher quality.
\end{abstract}

Keywords: color correction, information enhancement, multi-objective optimization, particle swarm optimization

\footnotetext{
*Corresponding Author

Email address: nmkwok@unsw.edu.au (N. M. Kwok)
} 


\section{Introduction}

Color images have been increasingly used in our daily life and in industrial applications. For viewing by a human viewer, color harmonization of the image is often desirable [1]. Raw images captured in digital cameras are thus frequently processed by computational algorithms to achieve an enhanced aesthetics effect [2]. In the context of industrial applications, the camera is a class of indispensable sensors providing valuable information for the operations of autonomous machines. For example, the three-dimensional shape of an object can be modeled by casting structured color lights on its surface [3]. A vision assisted system is also available in detecting pavement cracks [4]. In addition, vision-based automatic systems are also very popular in manufacturing processes [5].

Despite the above successful application examples, the quality of the captured image still plays a very important role that critically affects the performance of a vision-guided automatic system. One of the fundamental limitations lies on the difficulty in controlling the lighting conditions in the operation environments. For instance, the intensity of the illumination may be insufficient, be occluded, and most importantly, the illumination color may be uncontrolled. The cause of these problems could be interferences among illumination sources or gradual degradation due to aging. They are customarily classified as the color constancy problem that requires color correction [6] [7] as an embedded functionality in autonomous systems.

A possible approach to mitigate the adverse effects of uncontrollable illumination color is to estimate the illumination and then correct the color cast [8]. Within the available computational methods to correct the illumination color [9], there are several classes of algorithms for solving these problems. One of the classes is related to white balancing [10]. This method is based on the assumption that the brightest pixel in an image would correspond to white. However, in some environments, there may be no white objects and the algorithm may not perform as expected. Another possible approach in color correction is by the method of gamut mapping [11]. This method assumes that the color contained in an image is only a subset of all possible cases under all illuminations. A training set of images is obtained under some known conditions and a given image is then corrected according to the relationships derived from the training set. Also by making use of a similar principle, the correlation method was developed [12], to obtain the correlation between possible illuminations and objects. Then the most probable illumination color is estimated for an input image by the maximum correlation. Instead of using training images, a color correction approach was proposed in [13]. The correction operates on the hypothesis that the average edge difference in a scene is achromatic; hence, the color of the light source could be estimated from the deviations of the edge colors from the hypothesized values. However, enhancements of the edges in the image was not specifically considered.

There are other variations in tackling the color constancy problem. For example, color balancing of digital photographs are obtained by using simple image statistics [14]. Upon the acquisition of a knowledge of the illuminating color, the cast can be removed and produce a natural appearing resultant image. A similar approach also based on classification of the given image was reported in [15]. Specifically, an image is classified as taken either indoor or outdoor on the basis of observations and training data. Furthermore, an independently developed approach using a similar principle was presented in [16]. The given image is categorized into one of the groups containing 
various scene semantics and then different but canonical color correction algorithms are applied. An interesting approach was recently proposed that employs multiple image frames to correct color casting [17], whereby, the illumination color is estimated on the first captured frame and correction is conducted in subsequent frames on individual color channels. Further estimation based approaches can be found in [18] and [19]. However, it is generally difficult to obtain an exhaustive and complete priori distributions as required by the Bayesian type procedures.

In addition to the white point balancing method, methods based on gray world assumption are also effective approaches for color correction [20]. The principle of the gray world assumption stems from the hypothesis that the average color of a given image is achromatic and that the averages of the primary colors are close to each other that produces a gray shaded appearance. There are also methods that adopt both white point and gray world principles as the basis in their implementations. For example, a hybrid algorithm was developed in [21] that catered for local and global color corrections. Furthermore, a quadratic combination of these two assumptions was employed in [22] for color correction. In particular, the combination ensures that both white point and gray world assumptions are satisfied. In addition, other choices of assumptions had also been proposed in the literature. For example, in [23], the determination of illumination colors was dependent on the validity of assumptions on flatness of Lambertian object surfaces captured in an image and single lighting source.

Most developed methods for color correction that had been reported in the literature adjust the magnitude of the primary red, green and blue (RGB) color channels with appropriate gain factors. On the other hand, the determination of these factors may be different and depends on the particular method adopted. For example, the standard deviations of the color channels are used to find the gain factors [24]. The use of the standard deviation naturally provides a better description of the distribution of the color content that facilitates a better correction and removal of the color cast. In the work reported in [25], a subset of pixels is selected as white points based on the absolute difference to their corresponding mean values in the RGB channels. The multiplicative scaling factors for color channels adjustments are then calculated from this subset. An alternative method also using a subset of pixels was presented in [26], wherein, the white points are selected by converting the RGB channels into the YUV color space and thresholds are applied to classify white points. The RGB channel gains are then adjusted iteratively such that the white points are steered to converge to their gray color equivalents. A summary and evaluation of conventional and popular methods was given in [27] with the aim to quantify algorithms used in restoring faded photographs.

Recently, variations to the conventional gain adjustment methods have been reported in the literature. For instance, white balancing is conducted by stretching the color pixel distribution [28]. In a certain sense, the color channel gain adjustments are implemented in a non-linear manner. In parallel to the same philosophy, RGB channels were adjusted non-linearly using the gamma correction approach [29]. Particularly, this method is able to avoid display saturation which may be caused by gain factors larger than unity.

Although the methods appear in literature have produced satisfactory results in the context of color correction, the quality of an image may still be affected by other factors. Other developments in image contrast enhancement include the use of the gamma correction falling within the class of nonlinear power-law gain adjustments [30] and [31]. The contrast or information content also needs to be increased either for better view- 
ing or easier subsequent processing such as object identification and recognition. For instance, parameterized Bezier curves [32] were used as references in the adjustment. While enhancing image contrast, mean brightness was also considered as an important requirement [33]. Contrast enhancements could also be realized by hardware circuitries [34]. On the other hand one may tackle this problem from an optimization perspective. Specifically, proper color channel gain factors should be sought such that the resultant image satisfies both requirements for color cast removal and scene information recovery. Hence, a multi-objective optimization approach should be adopted.

With regard to multi-objective optimization, soft computing and intelligent computation algorithms are very suitable candidates to accomplish a desired optimization goal in solving image processing problems. For instance, a real coded genetic algorithm with a fuzzy clustering scheme was used in segmenting magnetic resonance images of the human brain [35]. Similarly, a non-dominated sorting genetic algorithm was adopted to optimize thresholds in image classification [36]. In addition to the genetic algorithm, there have been other nature inspired optimizations developed recently. Examples include the application of the artificial bee colony algorithm in segmenting synthetic aperture radar images [37]. The algorithm is able to account for image quality degradations due to the effect of speckle noise. Furthermore, the artificial ant colony algorithm was employed together with fuzzy sets to enhance the contrast of a high dynamic range color image [38]. A recent survey in the aforementioned research field can be found in [39].

Another popular nature inspired and agent based optimization approach is the particle swarm optimization (PSO) algorithm [40]. This algorithm simulates the motion of bird flocks that search for food. The inspiration is applied in solution schemes that search for optimal solutions where particles as candidate solutions are steered to travel through the parameter space. The advantages of PSO include its implementation simplicity and promising performance. Its control parameters, on the other hand, have been shown to be easily tuned and impose a minimal effect on the performance [41]. Improvements on the effectiveness of the PSO and its applications were reported in the literature. For example, a perturbed assignment of a lead particle was proposed in [42] accounting for possible traps in local optima. As with other intelligent computation algorithms, the PSO is also extendable to solve problems in multi-objective optimization problems [43]. With regard to image processing, the PSO has been successfully applied for image contrast enhancement [44] and in segmenting infrared images [45]. A further application in color image white balance has been recently reported in [46].

In this research, the problem of color correction is tackled in an unique perspective from the multi-objective optimization perspective taking into account the removal of illumination color cast and the maximal recovery of information contained in the image. The contribution of this paper is three folded. Firstly, the limitations on gray world and with patch based algorithms for color correction are revealed. In particular, the problem of over stretching of pixel magnitudes and the possibility of reductions in information content are illustrated. Secondly, color correction is posed as a non-linear gain adjustment process on the color channels using the gamma correction. This approach is able to mitigate the possibility of the resultant image saturating the display device. Thirdly, the PSO algorithm is enhanced with adapting to the need of searching for multi-objectives. A particle attraction and selection scheme is formulated that facilitates the construction of the Pareto front which ensures that solutions obtains are straightly non-dominating. A strategy in determining the final solution from the Pareto front is also suggested. 
The rest of the paper is organized as follows. In Section 2, the background and challenges in color correction based on the gray world assumption are presented. The multi-objective optimization process using the PSO is developed in Section 3. Experiments conducted using a collection of natural color images are described in Section 4 and results are discussed therein. Finally, a conclusion is drawn in Section 5.

\section{Color Correction Approaches}

Most digital cameras deliver color images in three color channels. These channels could be the primary red-green-blue (RGB) signals or other formats that are easily converted to the RGB color space equivalent. In this work, we assume that the image is available in the RGB format. Independent of the color representation, an image can be described by an image formation model [16]:

$$
f_{c}(\mathbf{x}) \propto \int_{\omega} \rho(\lambda) I(\lambda) S(\lambda) d \lambda
$$

where $f_{c}$ is the pixel value of a color channel $c \in\{R, G, B\}$, $\mathbf{x}$ is the location of the pixel in the image, $\omega$ is the range of the visible wavelength $\lambda$ in the electromagnetic spectrum, $\rho(\lambda)$ is the camera sensitivity, $I(\lambda)$ and $S(\lambda)$ are the illumination source and object surface reflectance respectively. From this model, it is evident that the color of the illumination source could affect the color of the pixel formed in the image.

The goal of color correction is to reconstruct the image, in terms of its color content, as if it was formed under a light source that is uniform across the visible spectrum $\omega$, or when the source color is white. The problem considered is ill-conditioned such that for an unknown light source, as it is difficult to obtain a correct reference of the color components. Hence, several assumptions are formulated when algorithms are implemented to conduct color correction. The two most popular candidates are, namely, the white patch and gray world assumptions [6]. The white patch based approach assumes that the brightest pixel in an image is white. The gray world assumption hypothesized that the average color magnitudes of the pixels would be equivalent to a gray tone. The latter assumption is more frequently justified in natural images where the image may contain a substantial amount of different colors.

\subsection{Gray World based Color Correction}

Consider a given image in the RGB format [29],

$$
\mathcal{I}=\left\{\mathbf{I}_{u v}\right\}, \mathbf{I}_{u v}=\left\{R_{u v}, G_{u v}, B_{u v}\right\},
$$

where $R_{u v}, G_{u v}, B_{u v}$ are the magnitudes of the appropriate color channels of the pixel while the coordinates at $(u, v)$ abide by the raster convention and are given by $u=$ $1, \cdots, U$ and $v=1, \cdots, V$ for an image of width $U$ and height $V$. Furthermore, the magnitudes of the color channels are confined below a maximum value corresponding to the common 8-bit representation up to $2^{8}-1=255$ levels, that is,

$$
R_{u v}, G_{u v}, B_{u v} \in[0,255]
$$


The conventional gray world color correction approach calculates the mean values of the RGB color channels and then finds two scale factors for the R- and B-channels respectively [20]. We have,

$$
\bar{\alpha}_{r}=\frac{\bar{G}}{\bar{R}}, \bar{\alpha}_{b}=\frac{\bar{G}}{\bar{B}}
$$

where

$$
\bar{R}=\frac{1}{U V} \sum_{u v} R_{u v}, \bar{G}=\frac{1}{U V} \sum_{u v} G_{u v}, \bar{B}=\frac{1}{U V} \sum_{u v} B_{u v} .
$$

The corrected image is obtained by linearly multiplying the R- and B-channels with their associated gain factors while the G-channel is left unchanged. That is,

$$
\tilde{R}_{u v}=\bar{\alpha}_{r} R_{u v}, \tilde{G}_{u v}=G_{u v}, \tilde{B}_{u v}=\bar{\alpha}_{b} B_{u v} .
$$

A test image together with the gray world corrected result is shown in Fig. 1 to illustrate the performance of this approach. It is noticed that the original image is highly cast in a yellowish tone while the corrected image has shown a removal of the dominated illumination color. For the purpose of removing color cast, the gray world based correction is performing satisfactorily.

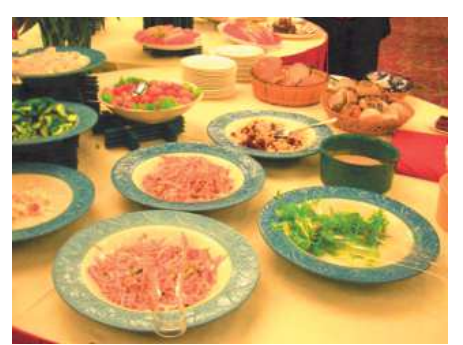

(a)

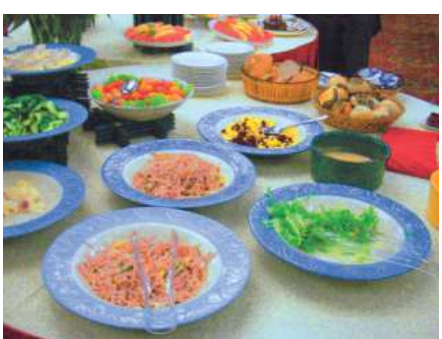

(b)

Figure 1: Gray world based color correction - Test Image 1: (a) original image, (b) gray world corrected image.

\subsection{Quadratic Color Correction based on Combined Gray World and White Patch}

Based on the argument that the brightest point in the image tends to reflect the color of the illumination source. The white patch algorithm was developed to normalize the maxima of the RGB channels to produce white patches in the resultant image [10]. The maxima of the individual channels are firstly obtained,

$$
\hat{R}=\max _{u v}\left\{R_{u v}\right\}, \hat{G}=\max _{u v}\left\{G_{u v}\right\}, \hat{B}=\max _{u v}\left\{B_{u v}\right\} .
$$

Then gain factors for the R- and B-channels are determined from

$$
\hat{\alpha}_{r}=\frac{\hat{G}}{\hat{R}}, \hat{\alpha}_{b}=\frac{\hat{G}}{\hat{B}} .
$$


Finally, the R- and B-channel magnitudes are modified accordingly while the G-channel is not altered. The color corrected image then becomes,

$$
\dot{R}_{u v}=\hat{\alpha}_{r} R_{u v}, \dot{G}_{u v}=G_{u v}, \dot{B}_{u v}=\hat{\alpha}_{b} B_{u v} .
$$

However, it has been revealed in the literature that white patch based color correction may suffer from the fact that white points may not appear sufficiently in an image to warrant the effectiveness of adjusting the pixels to the few white points. Furthermore, due to the simplicity in gray world based correction, a hybridization of these two assumptions have been made in the work reported in [22].

The concept proposed was motivated by the requirement that the processed color image should satisfy both the gray world and white patch assumptions. As presented in [10], this method of color correction is developed on the basis of formulating a quadratic function that represents the corrected image. For example, the corrected R-channel pixel magnitude is

$$
\hat{R}_{u v}=\mu_{r} R_{u v}^{2}+\nu_{r} R_{u v}
$$

where $\mu_{r}$ and $\nu_{r}$ are quadratic coefficients to be determined. In order to satisfy the gray world assumption, it is required that

$$
\mu_{r} \sum_{u v} R_{u v}^{2}+\nu_{r} \sum_{u v} R_{u v}=\bar{G}
$$

where $\bar{G}$ is the average of the G-channel pixel magnitudes. For the satisfaction of the white patch assumption, we need

$$
\max _{u v}\left\{R_{u v}\right\}=\max _{u v}\left\{G_{u v}\right\}=\hat{G}
$$

such that the maximum values of the $\mathrm{R}$ - and B-channels are aligned with the G-channel to achieve white balance. Hence, it requires

$$
\mu_{r} \max _{u v}\left\{R_{u v}^{2}\right\}+\nu_{r} \max _{u v}\left\{R_{u v}\right\}=\max _{u v}\left\{G_{u v}\right\}
$$

The coefficients $\mu_{r}$ and $\nu_{r}$ can be obtained from equations (11), (13), where

$$
\left[\begin{array}{l}
\mu_{r} \\
\nu_{r}
\end{array}\right]=\left[\begin{array}{cc}
\sum_{u v} R_{u v}^{2} & \sum_{u v} R_{u v} \\
\max _{u v}\left\{R_{u v}^{2}\right\} & \max _{u v}\left\{R_{u v}\right\}
\end{array}\right]^{-1}\left[\begin{array}{c}
\bar{G} \\
\hat{G}
\end{array}\right]
$$

These coefficients are then applied in equation (10) to obtain the correction for the pixel color. The B-channel can be corrected in the same manner; hence a color corrected image is obtained. An example image is shown in Fig. 2. In the regard of illumination color cast removal, the quadratic correction method is also considered effective.

\subsection{Evaluation of Multiplicative Color Correction Approaches}

The gray world and the quadratic based color correction methods, though using different ways to obtain the scaling factors, rely on multiplicative adjustment of the color channel magnitudes. Their effectiveness in color correction and information recovery are evaluated below. 


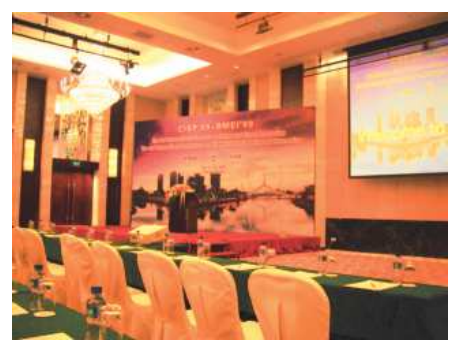

(a)

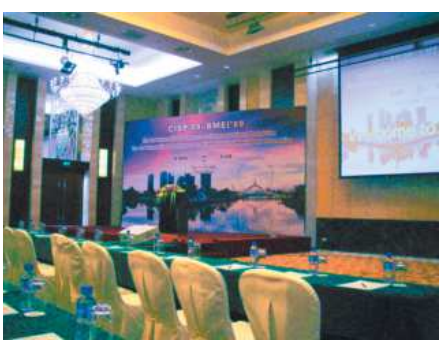

(b)

Figure 2: Quadratic based color correction - Test Image 2: (a) original image, (b) quadratic corrected image.

\subsubsection{Gray World based Color Correction}

We consider further the difference made in the context of the distribution of color pixels in the image. Figure 3 shows plots of histograms of the RGB channels of the original and corrected images corresponding to the image shown in Fig. 1.

It can be observed that in the original image histogram, the B-channel magnitude is small (peaked distributions at the low magnitude region) while the $\mathrm{R}$ - and G-channel magnitudes are large. The corresponding mean values of the colors are shown in diamond shaped markers. After color correction by multiplying with scaling factors $\bar{\alpha}_{r}$ and $\bar{\alpha}_{b}$, equation (6), it is seen that the magnitude of the R-channel is reduced while the Gchannel magnitude is not altered as expected. However, due to the multiplicative effect, the magnitude of the B-channel extended beyond the maximum value of $L=255$, see Fig. 3(b). This will cause a saturation effect in a display device and is not desirable.

A numerical assessment is further made that quantifies the degree accomplished in gray world based color correction. The color difference criterion builds upon the min-max concept and reflects the maximum absolute difference of individual channel mean values to the aggregated mean value. The assessment is given by

$$
\Delta=\max \{|\bar{R}-\bar{M}|,|\bar{G}-\bar{M}|,|\bar{B}-\bar{M}|\},
$$

where $\bar{M}=(\bar{R}+\bar{G}+\bar{B}) / 3$ is the aggregated mean color channel magnitude. As shown in Fig. 3 above, the original image has a color difference (labeled as "Diff") of 48.51 while that of the resultant image is close to zero. Hence, gray world based color correction is effective for this test image.

While the assumption of the gray world assumption is satisfied, an inspection on the information contents in the original and the corrected images is carried out. Here, the information contained in an image is defined as the value given by the Shannon entropy calculated from the gray-scale equivalent of the image. The color image is first converted to the gray-scale $Y$ by the YUV transformation [26], that is,

$$
Y=0.299 R+0.587 G+0.114 B \text {. }
$$

The probability that a channel magnitude falls in a particular level, $p_{i}$, is used to 
calculate the entropy given by

$$
H=-\sum_{i=0}^{255} p_{i} \log _{2} p_{i} \text { bits. }
$$

For the test image depicted in Fig. 1, the information contained in the original and corrected images are 7.72 and 7.71 bits respectively. It is observed that there is a slight reduction in the information content despite the satisfactory result in illumination color cast removal.

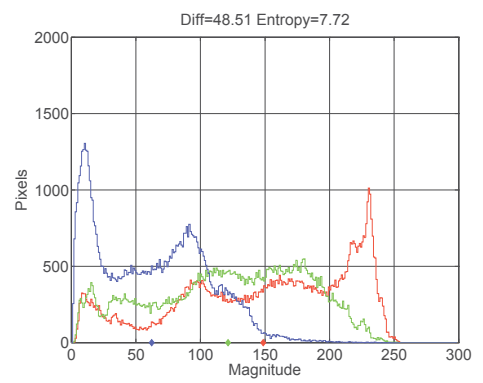

(a)

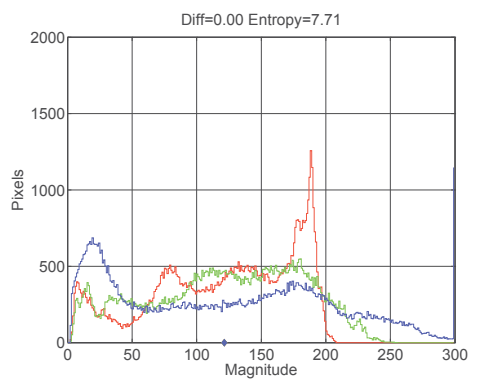

(b)

Figure 3: Histograms of the test image shown in Fig. 1, (a) original image, (b) gray world corrected image.

\subsubsection{Quadratic based Color Correction}

Similar assessments are made on the test image corrected by the quadratic method. The corresponding color distributions are shown in Fig. 4. It is seen that the color distributions also show a coincidence with the first test image in that there is a channel, here the B-channel, whose magnitude is less than the G-channel. After amplification by the scale factor $\alpha_{b}$, the B-channel pixels again saturate the display device.

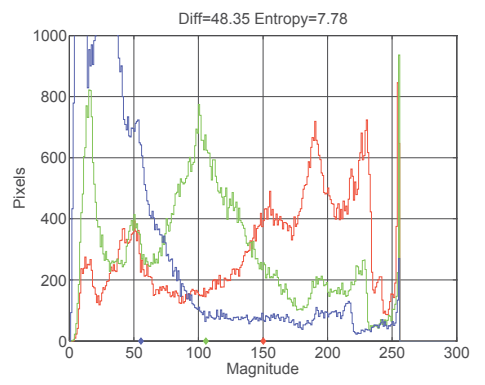

(a)

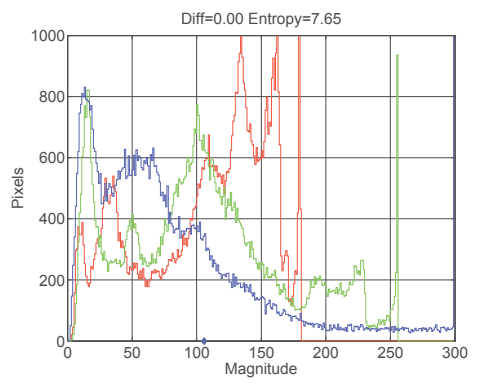

(b)

Figure 4: Histograms of the test image shown in Fig. 2, (a) original image, (b) corrected image by the quadratic method.

In the context of illumination cast removal, the resultant image has an aggregated difference of 48.35 and the quadratic color correction process is able to reduce it to almost 
zero. Hence, the correction is considered effective in this regard. For the information content, the original image contained 7.78 bits while the corrected image carries 7.65 bits of information. This is not a desirable result in the context of information recovery.

The observations from the evaluation indicate that illumination cast can be removed as expected due to the design of the method. However, the two procedures tend to introduced undesirable effects including display saturation and cases of reduction in information content carried in the color corrected image.

\subsection{Non-linear Color Correction}

The gray world based and the quadratic based color corrections described above rely on manipulating the color channel magnitudes of an image by a multiplicative scaling factor, see equations (6), (10) and (13). Hence, these approaches are classified as linear corrections. As discussed in the evaluation, there are possibilities that the color may be over multiplied and give rise to undesirable display saturations and consequently introduces a reduction of the information content. An alternative method adopting a non-linear adjustment principle, the gamma correction, was developed and reported in [29], focusing on color correction as well as preserving the brightness of the original image. A further extension of the concept is made in this research that simultaneously seeks the removal of the illumination cast and an increase of the information content where it will be posed as an optimization problem. Before presenting the development, the gamma correction based method is reviewed with its results illustrated here.

The gamma correction adjustment relies on raising the color channel magnitudes to the gamma coefficient as an exponent $\gamma_{r}$. We have,

$$
\hat{R}_{u v}=\check{R}_{u v}^{\gamma_{r}},
$$

where $\check{R}_{u v}=R_{u v} / 255$ is the normalized, for instance, R-channel magnitude.

Particularly, this approach for color channel adjustment possesses an advantageous feature that the magnitude of the adjusted color is bounded below unity if the original color magnitude is normalized. That is,

$$
\hat{R}_{u v} \in[0,1], \text { for all } \check{R}_{u v} \in[0,1] .
$$

As presented in [29], the gamma coefficient $\gamma_{r}$ is determined through a bi-sectional search process while keeping the brightness of the resultant image close to that of the original image. The result of test images are shown in figures 5 and 6 together with the plots of the corresponding histograms.

In figures 5(a) and 6(a), the color corrected images are depicted. The histograms shown in figures 5(b) and 6(b) indicate that there are no over-amplifications of any color channels. Hence, the gamma correction based non-linear color correction method is considered effective in mitigating the display saturation problem. Furthermore, it is noted that for the image shown in Fig. 5(a) that the information content has been increased from 7.72 to 7.75. Moreover, the information content for image shown in Fig. 6 (a) is only slightly decreased from 7.78 to 7.76 bits.

It can be seen that with the studied color correction schemes, the information contents are manipulatable while adjusting the color channel magnitudes. Therefore, an attempt is made that aims at correcting the image colors while simultaneously increases the information content. To this end, the problem is treated as a multi-objective optimization problem and its solution procedure will be developed and presented in the next section. 


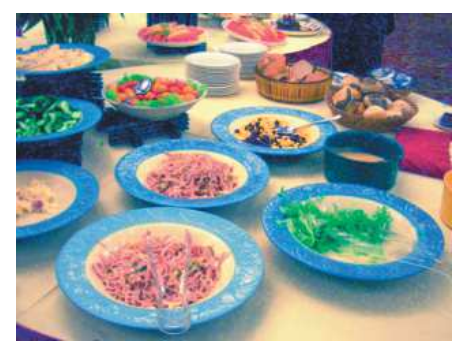

(a)

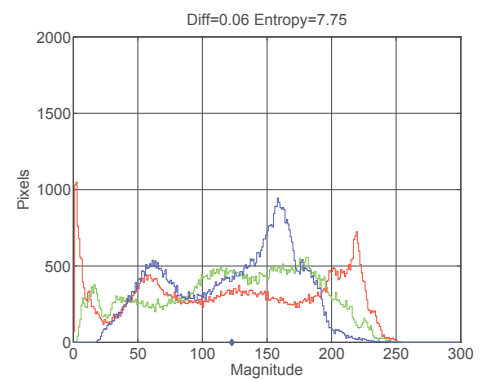

(b)

Figure 5: Non-linear color correction - Test image 1: (a) original image, (b) histogram of gamma corrected image.

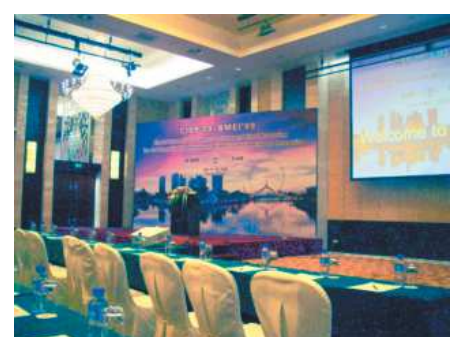

(a)

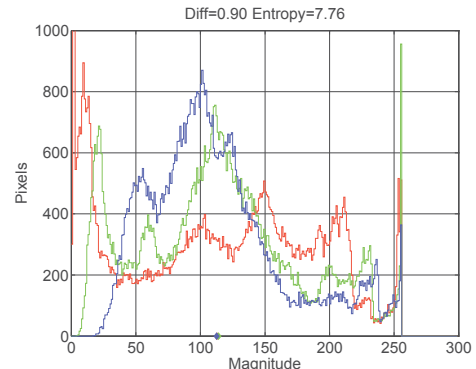

(b)

Figure 6: Non-linear color correction - Test image 2: (a) original image, (b) histogram of gamma corrected image.

\section{Multi-objective Optimization based Approach}

In many engineering applications when it is required to satisfy more than one design criterion in an optimal manner, multi-objective optimization methods have to be employed to provide feasible solutions to the problem. Moreover, in real-world problems, it is often difficult to have procedures that can lead to an analytical optimal solution. In these cases, nature inspired optimization algorithms are becoming attractive candidates [37], [38], [39], [42] and [44]. These methods include the artificial bee colony algorithm, artificial ant colony system, and the particle swarm optimization algorithm.

\subsection{Particle Swarm Optimization}

The particle swarm optimization (PSO) algorithm has obtained a lot of attention for its performance and implementation simplicity. The algorithm can also be extended to tackle multi-objective optimization problems.

\subsubsection{Principle of the PSO Algorithm}

In solving for the optimal solution of a problem, the potential solutions or decision variables are coded into a vector representation called a particle. This vector can be 
visualized as a point in a parameter space whose dimension corresponds to the size of the vector. It can be imagined that these particles were birds flying with certain velocities in the solution space while looking for food and escaping from predators. As a social metaphor, a bird with better food finding ability is treated as the leader and communicates its findings to other birds in the swarm. On the other hand, birds also rely on their individual experience to hunt for food. With these interactions, the whole bird flock gradually improves their food finding quality. In the seminal work of [40], this natural observation was transformed into an optimization algorithm.

\subsubsection{Description of the PSO Procedure}

The PSO iterative procedure can be described by the following expression,

$$
\begin{aligned}
& \mathbf{v}_{k+1}^{i}=\mathbf{w}^{i} \mathbf{v}_{k}^{i}+\mathbf{c}_{g}^{i}\left(\mathbf{g}_{\text {best }, k}-\mathbf{x}_{k}^{i}\right)+\mathbf{c}_{p}^{i}\left(\mathbf{p}_{\text {best }, k}^{i}-\mathbf{x}_{k}^{i}\right) \\
& \mathbf{x}_{k+1}^{i}=\mathbf{x}_{k}^{i}+\mathbf{v}_{k+1}^{i},
\end{aligned}
$$

where $\mathbf{x}^{i}$ is the $N$-dimensional particle position in the solution space, $\mathbf{v}^{i}$ is the velocity of the particle movement assuming a unity time step, $\mathbf{w}^{i}$ is the velocity control coefficient, $\mathbf{c}_{g}^{i}, \mathbf{c}_{p}^{i}$ are the gain control matrices, $\mathbf{g}_{\text {best }}$ is the global-best position, $\mathbf{p}_{\text {best }}^{i}$ is the position of a particular particle corresponding to its problem dependent best fitness obtained so far, subscript $k$ is the iteration index and superscript $i$ is the particle index.

At the start of the algorithm, the particle positions $\mathbf{x}_{0}^{i}$ are randomly assigned to cover the solution space [41]. These positions may be randomly distributed, given a pre-defined number of particles. In general, a small number of particles used reduces the computational load at the expense of extended iterations required to obtain the optimum solution. The initial particle velocity $\mathbf{v}_{0}^{i}$ can also be set randomly or simply assigned to zero. A problem dependent objective function is evaluated and an objective value or fitness is assigned to each particle. Based on the set of objective values, the particle having the lowest fitness (for a minimization problem) is taken as the global-best $\mathbf{g}_{\text {best }, 0}$. This set of initial objective values is denoted as the particle-best $\mathbf{p}_{\text {best }, 0}^{i}$. The velocity is then calculated using some random gain coefficients. The particle positions are updated and the procedure repeats. Finally, at the satisfaction of some termination criteria, the global-best particle is reported as the near-optimal solution to the problem.

\subsubsection{Pareto Optimality and Pareto Front}

In most engineering optimization problems, it is often required to accomplish optimal design objectives that are in conflict to each other. Thus, solutions have to be sought through Pareto optimality [43], and a brief description is given below.

Suppose it is required to solve the problem,

$$
\min \mathbf{f}(\mathbf{x}):=\left[f_{1}(\mathbf{x}), f_{2}(\mathbf{x}), \cdots, f_{k}(\mathbf{x})\right]
$$

subjected to decision variables $\mathbf{x}$ satisfying some constraints such that $\mathbf{x} \in \mathcal{X}$, where $\mathcal{X}$ is the feasible set of solutions. Due to the multi-objective nature, a single solution rarely occurs in practical problems. The concept of dominance and Pareto optimality are therefore adopted. The outcome from a multi-objective optimization routine would thus consists of solutions in the form of a Pareto front. The associated concept is best explained with the following definitions. 
Definition 1. Given two vectors $\mathbf{x}$ and $\mathbf{y}$, both real and of dimension $k$, that is, $\mathbf{x}, \mathbf{y} \in$ $\mathbb{R}^{k}$. It is defined that $\mathbf{x} \leq \mathbf{y}$, if $x_{i} \leq y_{i}$ for $i=1, \cdots, k$, and that $\mathbf{x}$ dominates $\mathbf{y},(\mathbf{x} \prec \mathbf{y})$, if $\mathbf{x} \leq \mathbf{y}$ and $\mathbf{x} \neq \mathbf{y}$.

Definition 2. A decision variable vector $\mathbf{x} \in \mathcal{X} \subset \mathbb{R}^{k}$ is non-dominated with respect to $\mathcal{X}$, if there does not exist a $\mathbf{x}^{*} \in \mathcal{X}$ such that $\mathbf{f}\left(\mathbf{x}^{*}\right) \prec \mathbf{f}(\mathbf{x})$.

Definition 3. A decision variable vector $\mathbf{x} \in \mathcal{F} \subset \mathbb{R}^{k}$, where $\mathcal{F}$ is the feasible region, is Pareto optimal if it is non-dominated with respect to $\mathcal{F}$.

Definition 4. The Pareto Set $\mathcal{P}^{*}$ is given by:

$$
\mathcal{P}^{*}=\{\mathbf{x} \in \mathcal{F} \mid \mathbf{x} \text { is Pareto optimal }\}
$$

Definition 5. The Pareto Front $\mathcal{P} \mathcal{F}^{*}$ is defined as:

$$
\mathcal{P} \mathcal{F}^{*}=\left\{\mathbf{f}(\mathbf{x}) \in \mathbb{R}^{k} \mid \mathbf{x} \in \mathcal{P}^{*}\right\}
$$

By making use of the above definitions of Pareto optimality and the construction of appropriate objective functions, the PSO can thus be adopted. It will be used in searching for satisfactory solutions for image color correction and enhancement simultaneously as a multi-objective optimization problem.

\subsection{Multi-objective Optimization for Color Correction and Information Enhancement}

As it has been revealed in Section 2, the quality of a color image needs to be improved where criteria including removal of illumination color cast and information content increment have to be met. In this paper, the PSO is used as a tool to accomplish this goal. In essence, to accommodate the requirement to satisfy the above mentioned two objectives, the conventional PSO described in Section 3.1.2 has to be modified. In particular, the Pareto front concept is adopted that converts the PSO into the multi-objective particle swarm optimization (MOPSO) algorithm.

The algorithm consists of the following major components including: particle initialization, iteration with objective evaluation, determination of the Pareto front and its management, particle update. The particle initialization and update stages follow the traditional implementation steps. The objective evaluation phase is problem dependent. In the current case, it is the color correction process and the assignment of the qualities in color correction and information gain. The manipulation of the Pareto front is purposefully developed in this research. The detailed steps are given below and the parameters used in the PSO are given in Table 1.

\subsubsection{Initialization}

The gamma values for each color channel adjustment are coded as a vector and is treated as a particle. That is,

$$
\mathbf{x}=\left[\begin{array}{lll}
\gamma_{r} & \gamma_{g} & \gamma_{b}
\end{array}\right]^{T}
$$

Note that iteration and particle indices are dropped for presentation convenience unless it is required in the context.

The number of particles included in the swarm $N$ and the number of generations $G$ are specified by the user, here $N=20, G=50$. The velocity control coefficient w will be 
Table 1: Parameters for PSO algorithm

\begin{tabular}{ll}
\hline Parameter & Value \\
\hline particle encoding & $\mathbf{x}=\left[\begin{array}{lll}\gamma_{r} & \gamma_{g} & \gamma_{b}\end{array}\right]^{T}$ \\
number of particles & 20 \\
number of iterations & 50 \\
inertia weight & $w=0.6$ \\
gain factors & $c_{g}, c_{p} \in[0,1]$ \\
\hline
\end{tabular}

a random number so that different values for each particle are used to ensure the ability to escape from local optima. The initial particle velocities are set to zero. The particle positions are sampled from an uniform distribution that gives $\gamma_{r}, \gamma_{g}, \gamma_{b} \in[0.5,2.0]$ in accordance to some preliminary experiments.

\subsubsection{Iteration}

The iteration stage forms the core of the PSO algorithm and can be described in Algorithm 1 below. The algorithm loops through each particle and each iteration. Color correction using the non-linear method is carried out, see (18). Then the objective values are found which consist of the color difference given in (15) and the entropy of the resultant image calculated from (17). After the operations for the particles, a Pareto front is generated using (27), see Section 3.2.3 below. Finally, the particle positions are updated as given by (20).

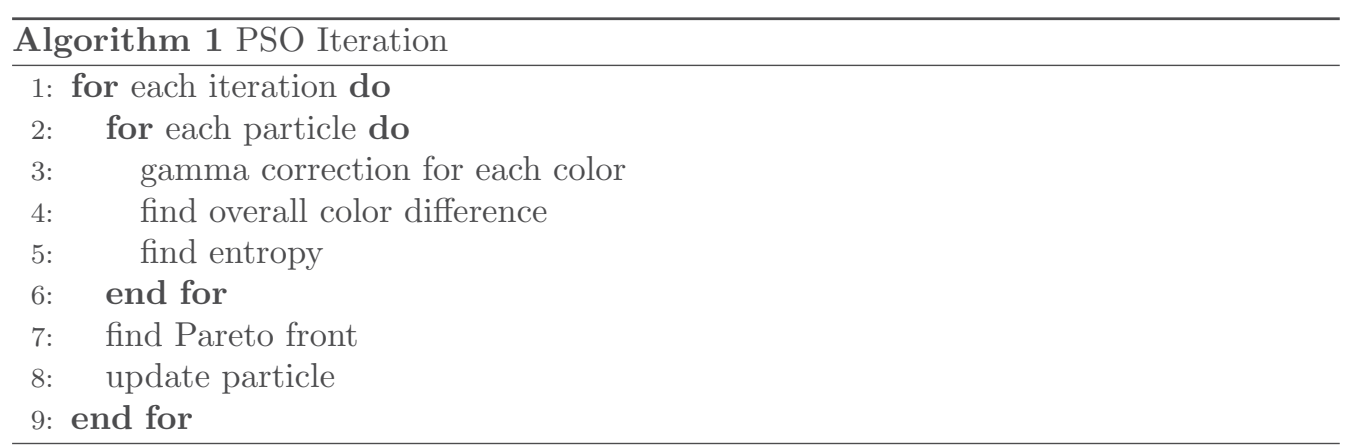

\subsubsection{Generation of the Pareto Front}

In order to account for the need to satisfy multiple objectives, the assignment of the global best particle $\mathbf{g}_{\text {best }}$ is modified. Let the objective values of an individual particle be $\Delta^{i}$ and $H^{i}$ in an iteration, say, the $k$ th iteration. The Pareto objective is

$$
K^{i}=\sum_{i}^{N}\left\{\left(\Delta^{i} \leq \Delta^{n}\right) \|\left(H^{i} \geq H^{n}\right)\right\}, n=1, \cdots, N
$$

where $\Delta^{i}, \Delta^{n}$ and $H^{i}, H^{n}$ are the objectives, namely, color differences and information contents, of all particles which are indicator values in [0,1] from the comparison and logical-or operator $(\|)$. The best particles are assigned as those non-dominating particles 
to form a temporary Pareto front [43] where there is no other particles that can better satisfy the objective. We have,

$$
\hat{\mathcal{P}}_{k}=\left\{\mathbf{x}^{j} \mid K^{i}=\max _{i}\left\{K^{i}\right\}\right\}, j=1, \cdots, J .
$$

Note that the number of particles $J$ in the set of temporary Pareto front is at most equal to the number of particles $N$. However, in most cases, it is less than the number of particles.

If it is the first iteration, then the particles in $\hat{\mathcal{P}}_{k}$ are assigned as on the Pareto front. If the current iteration is later than the first iteration, the currently selected particles are augmented to the previous front. Here an augmented front is formed as

$$
\tilde{\mathcal{P}}_{k}=\left\{\hat{\mathcal{P}}_{k}, \mathcal{P}_{k-1}\right\}
$$

where $\mathcal{P}_{k-1}$ is the final Pareto front in the previous $k-1$ th iteration. The Pareto objectives for the particles on the augmented front are rechecked to remove the dominated particles. That is, to check

$$
\tilde{K}^{q}=\sum\left\{\left(\Delta^{q} \leq \boldsymbol{\Delta}_{k-1}\right) \|\left(H^{q} \geq \mathbf{H}_{k-1}\right)\right\}, q=1, \cdots, Q,
$$

where $\boldsymbol{\Delta}_{k-1}$ and $\mathbf{H}_{k-1}$ are the objectives of particles on the previous Pareto front, and $Q$ is the number of aggregated particles on the Pareto front.

Those particles in the current and previous iterations that are being dominated are removed from the Pareto front. We now have the final Pareto front at the current $k$ th iteration as

$$
\mathcal{P}_{k}=\left\{\mathbf{x}^{m} \mid \tilde{K}^{q}=\max _{q}\left\{\tilde{K}^{q}\right\}\right\} .
$$

The particles on the latest refined Pareto front are employed to take the role of $\mathbf{g}_{b e s t, k}$ in subsequent PSO iterations. It is also noted that the number of particles that are located on the Pareto front may be less than or more than the number of particles in the swarm. This discrepancy in cardinality is problem dependent and related to the objective space landscape. In order to allow for the PSO algorithm implementation with the same number of $\mathbf{g}_{\text {best }}$ particles as particles in the swarm, the particles on the Pareto front are sampled with equal probability to generate the set of global $\mathbf{g}_{\text {best }}$ particles. We have,

$$
\mathbf{g}_{b e s t, k}^{i} \longleftarrow \mathcal{P}_{k}^{m}, i=1, \cdots, N,
$$

where the mapping $i \longleftarrow m$ is drawn randomly from a uniform distribution. A summary of the stages involved in the generation of the multi-objective Pareto front is given in Algorithm 2 below.

\subsubsection{Particle Update}

Particle updates as implemented here basically follow the conventional PSO algorithm structure as given in (20) [40]-[45]. In addition, a simplification is made that the update according to the self-experience term $c_{p}^{i}\left(\mathbf{p}_{\text {best }}^{i}-\mathbf{x}^{i}\right)$ is not implemented. This simplification is justified by the fact that in the multi-objective scheme, the global best particles now becomes a diversified set instead of a single particle. Hence, a sufficient degree in search randomness is maintained. After the update, particles are fed to the next iteration to search for a better solution until the maximum number of iterations is reached. 


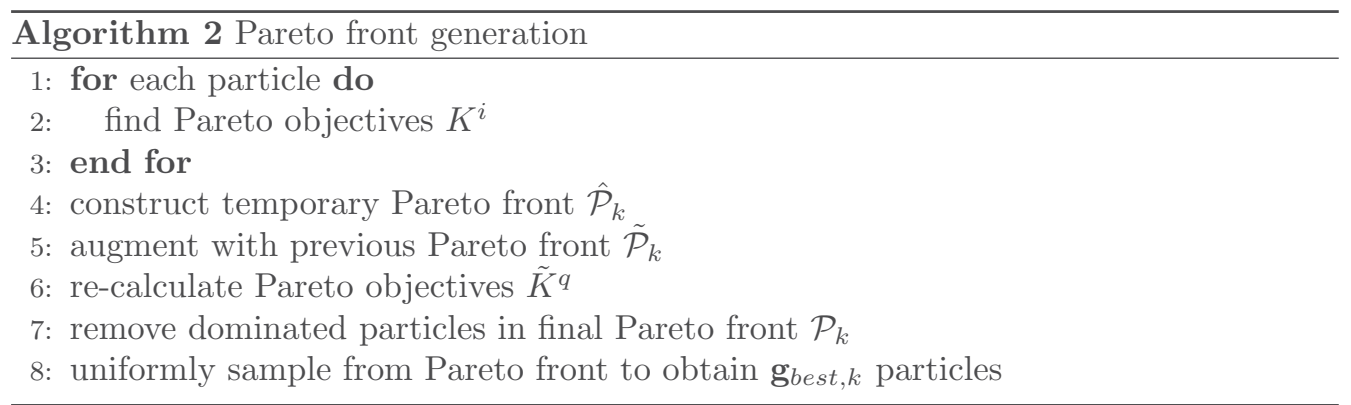

\subsubsection{Selection from the Pareto Front}

According to the principle of multi-objective optimization, particles on the Pareto front are all optimal solutions in the sense that alternations in the decision variables, the gamma values, would make the corresponding objectives degrade. The selection of a single solution from the Pareto front is still an open question unless there is a higher hierarchy of criteria imposed by the user. Examples of plots of the objective space illustrating the Pareto front are presented below to demonstrate the challenge.

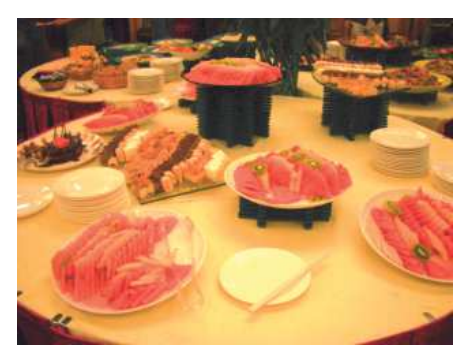

(a)

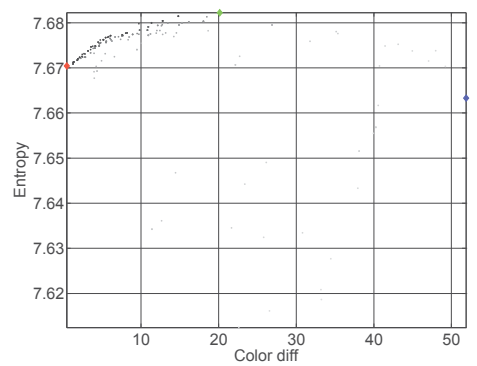

(b)

Figure 7: Pareto fronts of color corrected images - Test image 3: (a) original image, (b) Pareto front

In figures $7(\mathrm{~b})$ and $8(\mathrm{~b})$, the objective values of the original values are shown as a blue dot and they both appear at the right or bottom-right corner of the objective portrait. In the portrait, the horizontal axis corresponds to the discrepancy to the satisfaction of the gray world assumption. A low color difference indicates a better accomplishment of the objective. The vertical axis corresponds to the information content and a higher value indicates larger information content contained in the color corrected image. The objectives of particles on the Pareto front are shown in black dots while gray dots denote objectives of previous PSO iterations. It is observed that both corrections result in improving the objectives as indicated by the concentration of dots at the top-left region of the objective space.

In this research, our aim is to seek a set of gamma correction values such that the color corrected image would possess a close satisfaction of the gray world assumption and an increase in the information conveyed from the scene. However, it is anticipated that compromises in both objectives are needed for some images. Two test images and their associated objective space plots are shown in figures 9 and 10 . 


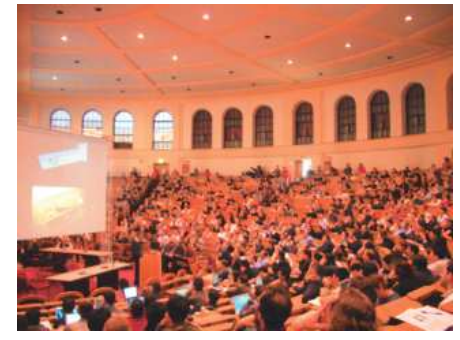

(a)

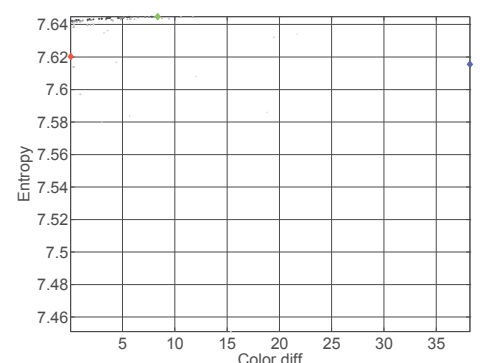

(b)

Figure 8: Pareto fronts of color corrected images - Test image 4: (b) original image, (b) Pareto front

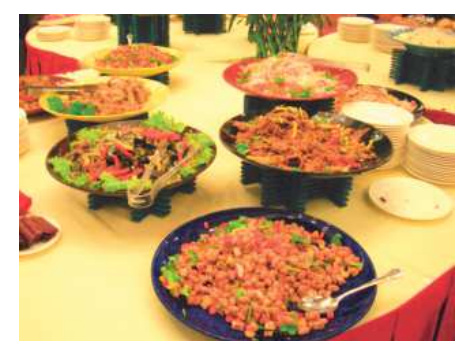

(a)

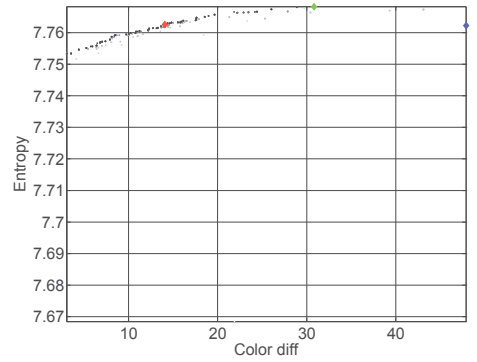

(b)

Figure 9: Pareto fronts of color corrected images - Test image 5: (a) original image, (b) Pareto front

For Test Image 5 with the objective space depicted in Fig. 9(b), while striving for minimum color difference to the gray world, the information content of the resultant image would be reduced. On the other hand, as shown in Fig. 10(b) for Test Image 6, if a higher information content is sought, an increase in color difference is unavoidable. Further test images shown in figures 11 and 12, are also included to demonstrate the distribution of Pareto fronts for images with different color casts such as bluish and grayish tones.

When the effectiveness of the accomplishment of the dual objectives depends on the characteristics of the input image, a Pareto front selection scheme is proposed. Here, we propose to provide the user with two resultant images according to the two objectives. The resultant image selected with minimum color difference to the gray world assumption is subject to the constraint that the associated color difference is no larger than the original image. That is,

$$
\mathbf{x}^{c}=\left\{\mathbf{x}^{i} \mid \Delta^{i} \leq \Delta_{0}\right\}
$$

where $\mathbf{x}^{c}$ is the selected particle of gamma values, and $\Delta_{0}$ is the color difference of the original image.

Similarly, the image select for maximum information conveyed is subject to no degradation of the gray world assumption that the resultant information is not less than the original image. We have,

$$
\mathbf{x}^{h}=\left\{\mathbf{x}^{i} \mid H^{i} \geq H_{0}\right\}
$$




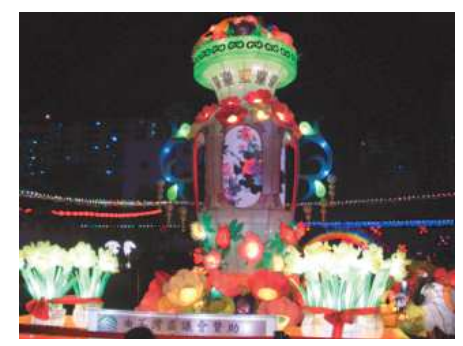

(a)

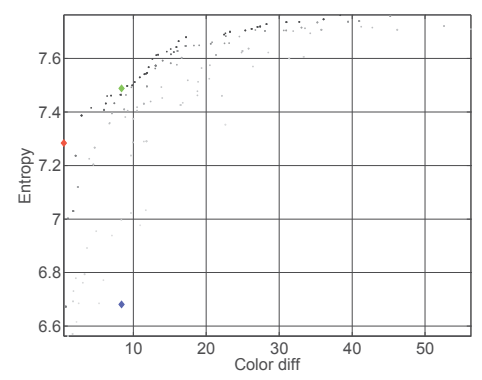

(b)

Figure 10: Pareto fronts of color corrected images - Test image 6: (a) original image, (b) Pareto front

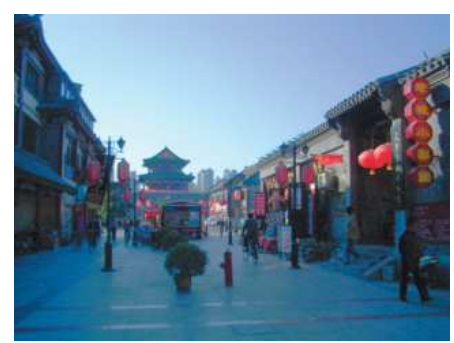

(a)

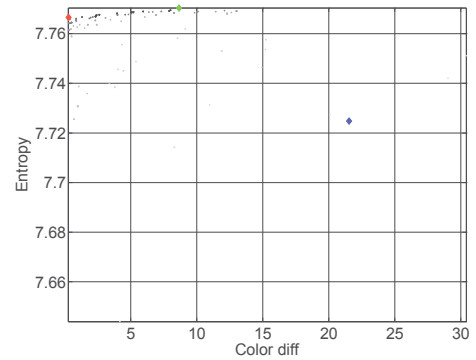

(b)

Figure 11: Pareto fronts of color corrected images - Test image 7: (a) original image, (b) Pareto front

where $\mathbf{x}^{h}$ is the selected particle of gamma values, and $H_{0}$ is the information content of the original image.

As shown with the test images in figures 7 to 12 , red dots denote selections for minimum color difference while green dots denote selections for maximum information content. The input images are shown in Fig. 13 and corresponding color corrected images are shown below in Fig. 14. For images satisfying the information based objective, the selected resultant images are shown in Fig. 15. As indicated by the numerical values of the objectives found in the objective portrait, the selected images subject to the two criteria have illustrated the trade-off made in choosing a single solution from a multiobjective optimization problem.

\section{Results}

In addition to the example results given in the above section, a collection of 40 color images taken under different illumination conditions are used in further investigations. There had been performance measures reported in the literature based on the availability of ground-truth references, for instance, the work in [47] and [48] had adopted the Euclidian distance and color vector angular errors. However, since references cannot be easily obtained for all conditions in natural scenes the application of references measures may find difficulties in practice. For the purpose of this work, we assess the effectiveness 


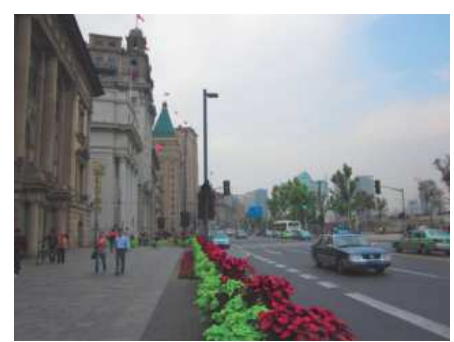

(a)

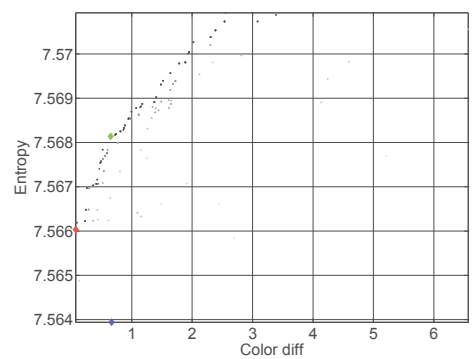

(b)

Figure 12: Pareto fronts of color corrected images - Test image 8: (a) original image, (b) Pareto front

of the proposed method by comparing the color differences and the information contents of the original and resultant images.

Scatter plots are depicted in Fig. 16. For assessment on the satisfaction of the gray world assumption, the horizontal axis represents the values of the original images while the vertical axis represents the resultant images. It is observed that all indicators are located below the diagonal line, in Fig. 16(a), indicating that there are reductions in the color difference in all test images. With regard to the information or entropy resulting from the color corrected images, most of them are above the diagonal line. This indicates that information gains are made in a majority of test images. Particularly, only those resulted from the multi-objective optimization processes, shown in magenta and red circles, produce increments.

A further illustration is provided using plots of histograms for the two objectives using the color correction methods described above, see Fig. 17. For the color difference criterion, all changes are positive indicating the effectiveness of all methods in this regard as confirmed in Fig. 17(a). On the other hand, with the information gain criterion as depicted in 17(b), some results do not have a positive gain. This is particular true for the gray world and quadratic methods shown in green and cyan curves.

A collection of the result statistics are depicted as box-plots in Fig. 18. For color difference assessment, we see that there are significant improvements by the gray world and quadratic methods. Approaches based on gamma correction and the PSO multi-objective selecting best color differences produce noticeable improvements, see Fig. 18(a). The multi-objective selection based on maximum information produces larger color differences. However, this is expected as a trade-off for information gain. Notwithstanding this insufficiency, the overall color differences are much lower than those from the original images. For the information criterion, the multi-objective based methods produce both higher information gain as compared to the original images and other methods as depicted in Fig. 18(b). In particular, it is observed that the gray world and quadratic methods give rise to reductions in the information content which may not be desirable.

Further resultant images illustrating the effectiveness of the proposed multi-objective approach for color correction are shown below. In Fig. 19, the original image is severely color biased in the yellowish tone due to an indoor light source with a warm color temperature. The result shown in Fig. 19(e) is corrected to achieve a color difference of 0.81 from the original image carrying a color difference of 51.88. It is observed that results 
from the canonical gray world multiplicative correction is biased towards a bluish tone while the quadratic correction method produced only an intermediate correction as expected. The gamma based adjustment method gives a result that is close to the optimal but its information content is slightly reduced.

The color corrected image with the largest improvement in information content is shown in Fig. 20. The resultant image using the gamma values so selected from the Pareto front is illustrated in Fig. 20(f). The information content increased from 6.68 in the original image to 7.55 in the color corrected image. This test image was captured in an outdoor scene at night where only artificial lighting is available. It is observed that only results from the proposed MOPSO method are performing well in the context of information content increment. In particular, the image corrected by gamma values selected on the Pareto front is able to expose the buildings in the background. This is because of the fact that the gamma values were selected using the maximum information criterion.

\section{Conclusion}

This paper has presented evaluations and improvements of methods for image color correction aiming at mitigating the effect of illumination color casts together with gains in the information contained in the image. Limitations of methods based on linear multiplicative color channel adjustments are revealed. It is shown that the gray world and quadratic approaches may not perform well if the criterion of information gain is taken into consideration. Drawbacks including display saturation and reduction in the information content conveyed in an image are also observed. Non-linear adjustment using the gamma correction principle is proposed and its performance investigated. It can be seen that display saturation is also mitigated. Further improvements have been made to satisfy both objectives of color correction and information gain when the task is posed as a multi-objective optimization problem. A particle swarm optimization procedure is develop to incorporate improvements in tackling the multi-objective optimization challenge. A new method to generate and handle the Pareto front is then proposed and shown to be effective. A selection scheme in producing final results is suggested to guarantee the improvement of the resultant images in at least any one of the criteria. Tests from natural color images are conducted and results have illustrated the effectiveness of the proposed PSO based multi-objective optimization method in color corrections and enhancement.

\section{References}

[1] D. Cohen-Or, O. Sorkine, R. Gal, T. Leyvand, Y. Q. Xu, Color harmonization, in: Proc. 2006 ACM Siggraph, 2006, pp. 624-630.

[2] L. C. Ou, M. R. Luo, A color harmony model for two-colour combinations, Color Research and Applications 31(3) (2006) 191-204.

[3] S. Y. Chen, Y. F. Li, J. Zhang, Vision processing for realtime 3-d data acquisition based on coded structured light, IEEE Trans. on Image Processing 17(2) (2008) 167-176.

[4] J. H. Lee, H. S. Yoo, Y. S. Kim, J. B. Lee, M. Y. Cho, The development of a machine vision-assisted teleoperated pavement crack sealer, Automation in Construction 15 (2006) 616-626.

[5] Y. Chen, M. A. jafari, Vision-based online process control in manufacturing applications, IEEE Trans. on Automation Science and Engineering 5(1) (2008) 140-153. 
[6] K. Barnard, V. Cardei, B. Funt, A comparison of computational color constancy algorithms - part i: methodology and experiments with synthesized data, IEEE Trans. on Image Processing 11(9) (2002) 972-983.

[7] K. Barnard, V. Cardei, B. Funt, A comparison of computational color constancy algorithms - part ii: experiments with image data, IEEE Trans. on Image Processing 11(9) (2002) 985-996.

[8] S. D. Hordley, Scene illuminant estimatin: past, present, and future, Color Research and Application 31(4) (2008) 303-314.

[9] V. Agarwal, B. R. Abidi, A. Koschan, M. A. Abidi, An overview of color constancy algorithms, Journal of Pattern Recognition Research 1 (2006) 42-54.

[10] E. Y. Lam, G. S. K. Fung, Automatic white balancing in digital photography, in: Single-sensor Imaging: Methods and Applications for Digital Camera, CRC Press, 2009, pp. 267-294.

[11] G. Finlayson, S. Hordley, Improving gamut mapping color constancy, IEEE Trans. on Image Processing 9(10) (2000) 1774-1783.

[12] G. D. Finlayson, S. D. Hordley, P. M. Hubel, Color by correlation: a simple, unifying framework for color constancy, IEEE Trans. on Pattern Analysis and Machine Intelligence 23(11) (2001) 12091221 .

[13] J. van de Weijer, T. Gevers, A. Gijsenij, Edge-based color constancy, IEEE Trans. on Image Processing 16(9) (2007) 2207-2214.

[14] F. Gasparini, R. Schettini, Color balancing of digital photos using simple image statistics, Pattern Recognition 37 (2004) 1201-1217.

[15] S. Bianco, G. Ciocca, C. Cusano, R. Schettini, Improving color constancy using indoor-outdoor image classification, IEEE Trans. on Image Processing 17(12) (2008) 2381-2392.

[16] A. Gijsenij, T. Gevers, Color constancy using natural image statistics and scene senantics, IEEE Trans. on Pattern Analysis and Machine Intelligence 33(4) (2011) 687-698.

[17] R. C. Bilcu, Multiframe auto white balance, IEEE Signal Processing Letters 18(3) (2011) $165-168$.

[18] D. H. Brainard, W. T. Freeman, Bayesian color constancy, Journal of the Optical Society of America - A 14 (1997) 1393-1411.

[19] M. D'Zmura, G. Iverson, B. Singer, Probabilistic color constancy, in: Geometric Representations of Perceptual Phenomena, Lawrence Erlbaum Assoc., 1995, pp. 187-202.

[20] H. Kawamura, S. Yonemura, J. Ohya, N. Matsuura, Illuminant color estimation by hue categorization based on gray world assumption, in: Proc. of SPIE-ST\&T Electronic Imaging, 2011, pp. $787312-1$

[21] A. Rizzi, C. Gatta, D. Marini, A new algorithm for unsupervised global and local color correction, Pattern Recognition Letters 24 (2003) 1663-1677.

[22] E. Y. Lam, Combining gray world and retinex theory for automatic white balance in digital photography, in: Proc. 9th Intl. Symposium on Consumer Electronics, 2005, pp. 134-139.

[23] D. A. Forsyth, A novel algorithm for color constancy, International Journal of Computer Vision 5 (1) (1990) 5-36.

[24] H. K. Lam, O. C. Au, C. W. Wong, Automatic white balancing using standard deviation of rgb components, in: Proc. IEEE Intl. Symposium on Circuits and Systems, 2004, pp. 921-924.

[25] C. C. Weng, H. Chen, C. S. Fuh, A novel automatic white balance method for digital still cameras, in: Proc. IEEE Intl. Symposium on Circuits and Systems, 2005, pp. 3801-3804.

[26] J. Hou, Y. Chang, J. Wang, X. Wei, Robust automatic white balance algorithm using gray color points in images, IEEE Trans. on Comsumer Electronics 52(2) (2006) 541-546.

[27] D. Nikitenko, M. Wirth, K. Trudel, White-balancing algorithms in colour photograph restoration, in: Proc. IEEE Intl. Conference on Systems, Man and Cybernetics, 2007, pp. 1037-1042.

[28] S. Wang, Y. Zhang, P. Peng, F. Zhou, Fast automatic white balancing method by color histogram stretching, in: Proc. 4th Intl. Congress on Image and Signal Processing, 2011, pp. 993-997.

[29] N. M. Kwok, D. Wang, X. Jia, S. Y. Chen, G. Fang, Q. P. Ha, Gray world based color correction and intensity preservation for image enhancement, in: Proc. 2011 4th Intl. Congress on Image and Signal Processing, Shanghai, China, 2011, pp. 1008-1012.

[30] S. C. Huang, F. C. Cheng, Y. S. Chiu, Efficient contrast enhancement using adaptive gamma correction with weighting distribution, IEEE Transactions on Image Processing 9 (1) (2013) 10321041.

[31] F. C. C. Y. S. Chiu, S. C. Huang, Efficient contrast enhancement using adaptive gamma correction and cumulative intensity distribution, in: Proc. IEEE International Conference on Systems, Man, and Cybernetics (IEEE SMC 2011), 2011, pp. 2946-2950.

[32] F. C. Cheng, S. C. Huang, Efficient histogram modification using bilateral bezier curve for the contrast enhancement, IEEE/OSA Journal of Display Technology 9 (1) (2013) 44-50. 
[33] S. C. Huang, C. H. Yeh, Image contrast enhancement for preserving mean brightness without losing image features, Engineering Applications of Artificial Intelligence 26 (2013) 1487-1492.

[34] W. C. Chen, S. C. Huang, T. Y. Lee, An efficient reconfigurable architecture design and implementation of image contrast enhancement algorithm, in: Proc. IEEE International Conference on Embedded Software and Systems (IEEE ICESS 2012), 2012, pp. 1741-1747.

[35] A. Mukhopadhyay, U. Maulik, A multiobjective approach to $\mathrm{mr}$ brain image segmentation, Applied Soft Computing 11 (2011) 872-880.

[36] A. Nakib, H. Oulhadj, P. Siarry, Image thresholding based on pareto multiobjective optimization, Engineering Applications of multiobjective optimization 23 (2010) 313-320.

[37] M. Ma, J. Liang, M. Guo, Y. Fan, Y. Yin, Sar image segmentation based on artificial bee colony algorithm, Applied Soft Computing 11 (2011) 5205-5214.

[38] O. M. Verma, P. Kumar, M. Hanmandlu, S. Chhabra, High dynamic range optimal fuzzy color image enhancement using artificial ant colony system, Applied Soft Computing 12 (2012) 394-404.

[39] C. Bong, M. Rajeswari, Multi-objective nature-inspired clustering and classification techniques for image segmentation, Applied Soft Computing 11 (2011) 3271-3282.

[40] J. Kennedy, R. Eberhart, Particle swarm optimization, in: Proc. IEEE Intl. Conference on Neural Networks, Perth, Australia, 1995, pp. 1942-1948.

[41] N. M. Kwok, D. K. Liu, K. C. Tan, Q. P. Ha, An empirical study on the settings of control coefficients in particle swarm optimization, in: Proc. 2006 IEEE Congress on Evolutionary Computing, Vancouver, BC, Canada, 2006, pp. 823-830.

[42] X. Zhao, A perturbed particle swarm algorithm for numerical optimization, Applied Soft Computing 10 (2010) 119-124.

[43] M. Reyes-Sierra, C. C. C. Ceollo, Multi-objective particle swarm optimizers: a survey of the stateof-the-art, International Journal of Computational Intelligence Research 2(3) (2006) 287-308.

[44] N. M. Kwok, Q. P. Ha, D. K. Liu, G. Fang, Contrast enhancement and intensity preservation for gray-level images using multiobjective particle swarm optimization, IEEE Trans. on Automation Science and Engineering 6(1) (2009) 145-155.

[45] F. Du, W. Shi, L. Chen, Y. Deng, Z. Zhu, Infrared image segmentation with 2-d maximum entropy method based on particle swarm optimization (PSO), Pattern Recognition Letters 26 (2005) 597603.

[46] C. Chen, S. Lin, Formulating and solving a class of optimization problems for high-performance gray world automatic white balance, Applied Soft Computing 11 (2011) 523-533.

[47] S. D. Hordley, G. D. Finlayson, Re-evaluation of color constancy algorithm performance, Journal of the Optical Society of America - A 23 (5) (2006) 1008-1020.

[48] A. Gijsenij, T. Gevers, M. Lucassen, A perceptual analysis of distance measures for color constancy algorithms, Journal of the Optical Society of America - A 26 (10) (2009) 2243-2256. 


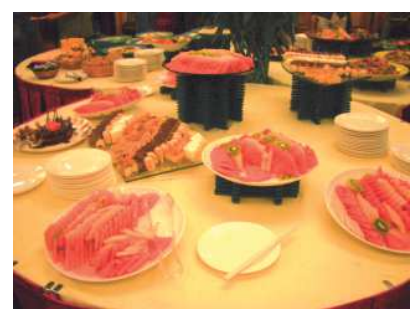

(a)

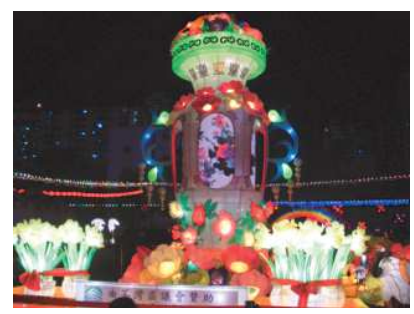

(d)

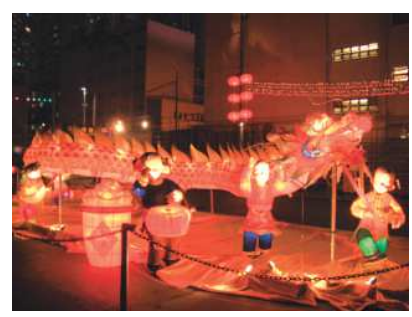

(g)

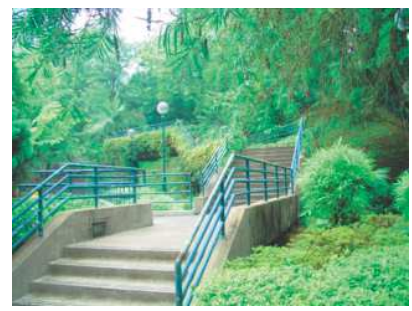

(j)

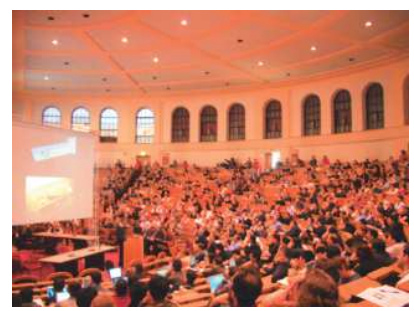

(b)

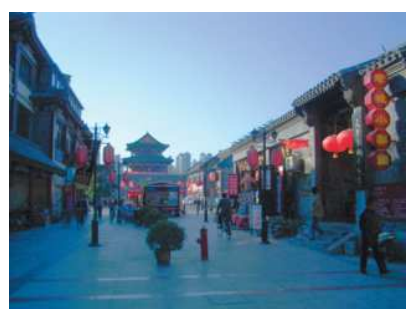

(e)

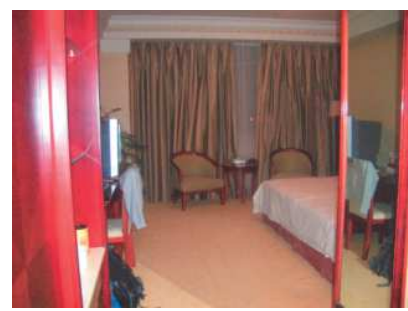

(h)

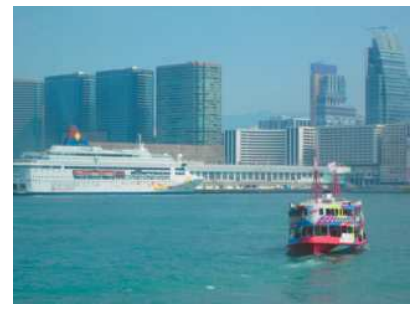

(k)

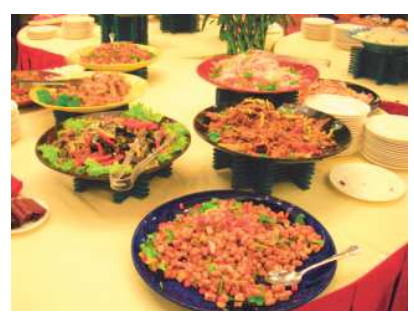

(c)

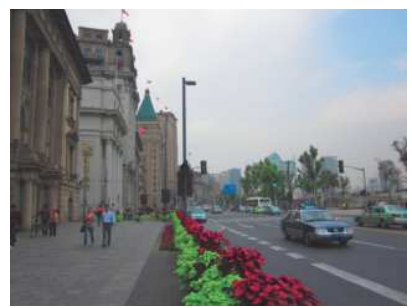

(f)

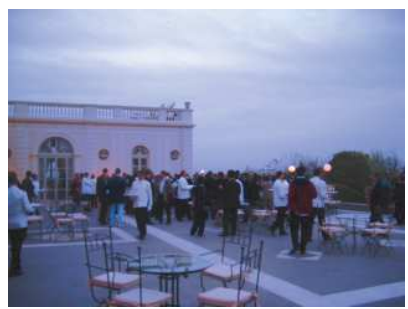

(i)

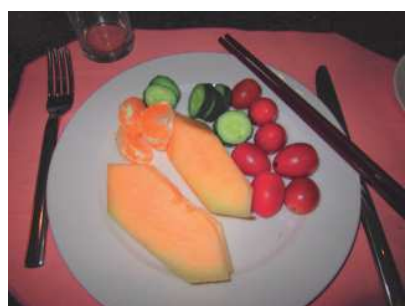

(1)

Figure 13: Input test images: (a) test image 3, (b) test image 4, (c) test image 5, (a) test image 6, (e) test image 7, (f) test image $8,(\mathrm{~g})$ test image $9,(\mathrm{~h})$ test image $10,(\mathrm{i})$ test image $11,(\mathrm{j})$ test image 12 (k) test image 13, (l) test image 14 . 


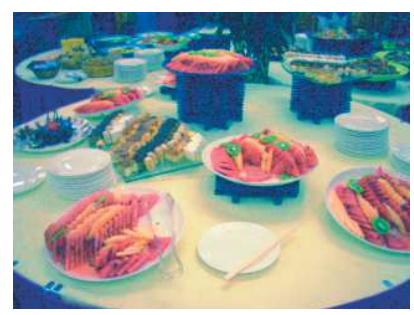

(a)

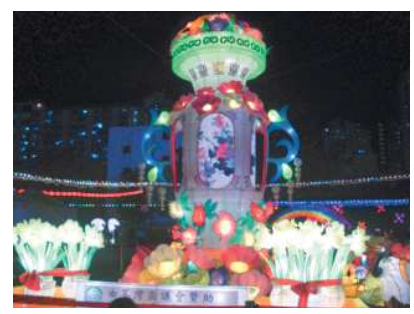

(d)

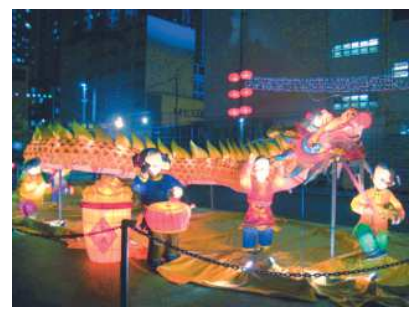

(g)

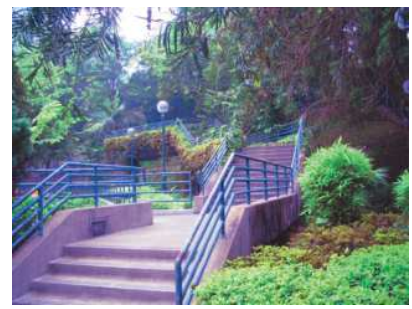

(j)

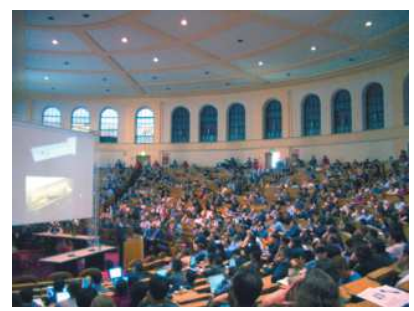

(b)

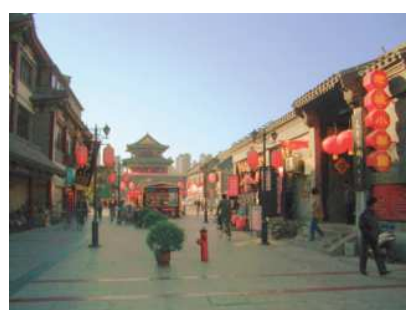

(e)

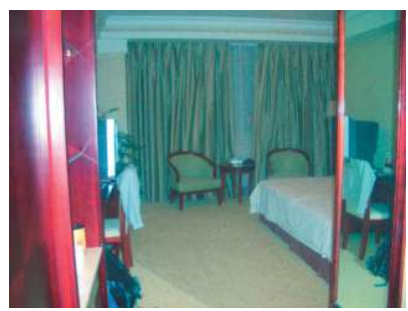

(h)

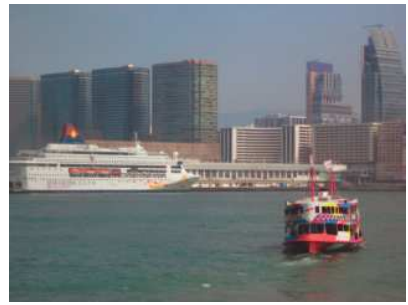

(k)

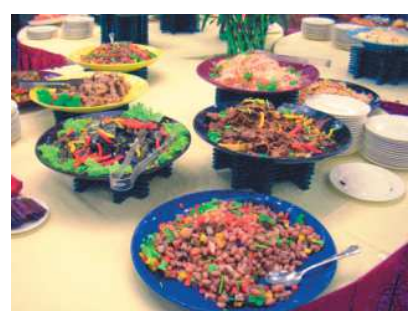

(c)

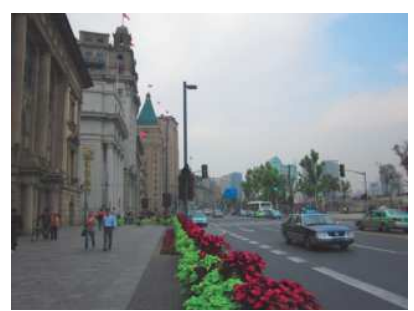

(f)

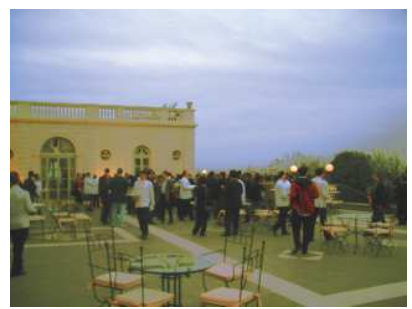

(i)

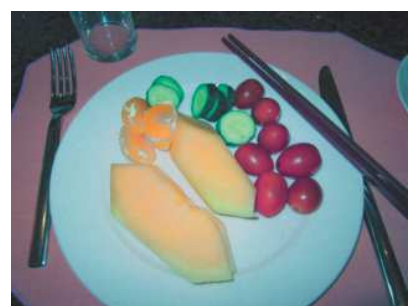

(1)

Figure 14: Color corrected images selected for minimum color difference: (a) test image 3, (b) test image 4, (c) test image 5, (d) test image 6, (e) test image 7, (f) test image 8, (g) test image 9, (h) test image 10, (i) test image 11, (j) test image 12, (k) test image 13, (l) test image 14. 


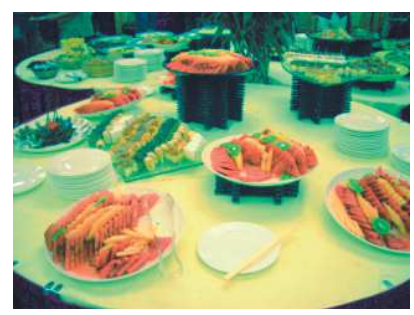

(a)

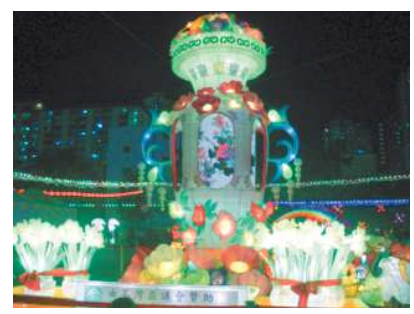

(d)

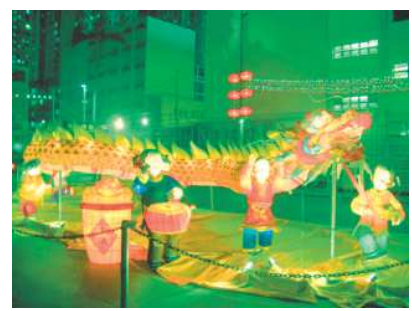

(g)

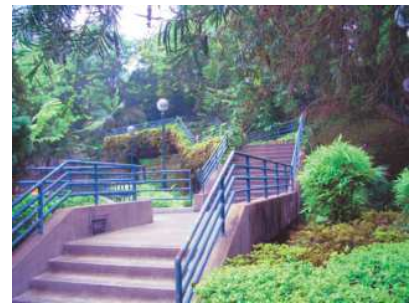

(j)

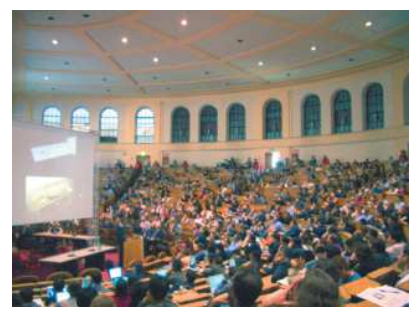

(b)

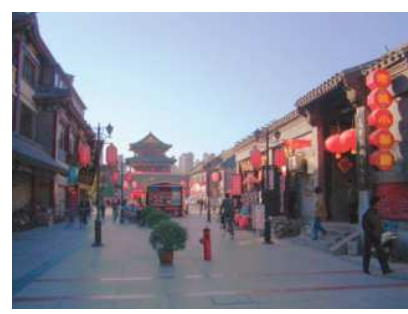

(e)

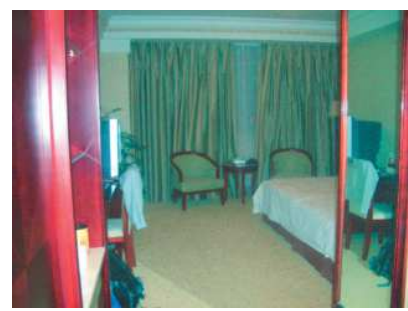

(h)

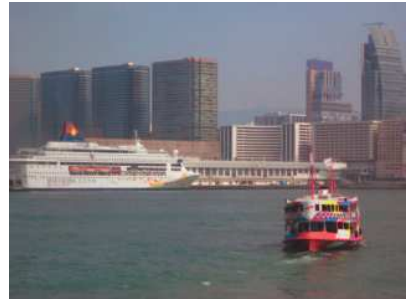

(k)

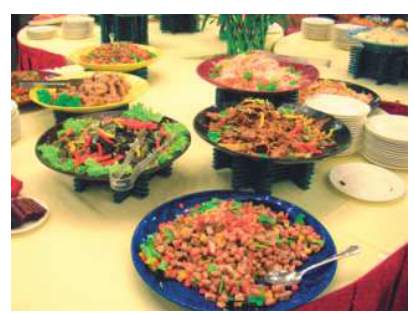

(c)

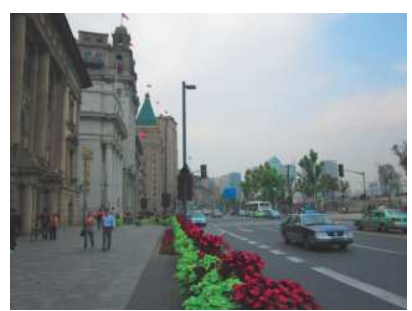

(f)

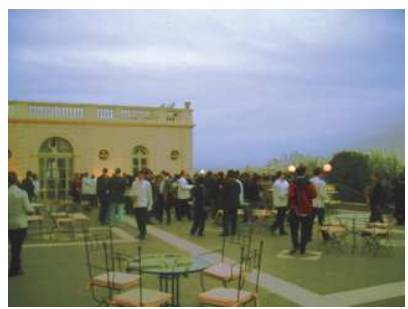

(i)

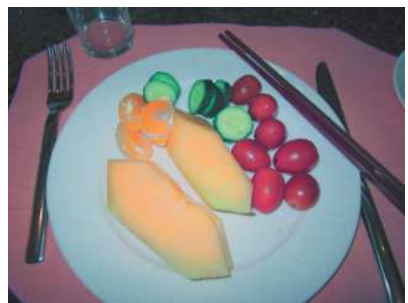

(1)

Figure 15: Color corrected images selected for maximum information gain: (a) test image 3, (b) test image 4, (c) test image 5, (d) test image 6, (e) test image 7, (f) test image 8, (g) test image 9, (h) test image 10, (i) test image $11,(\mathrm{j})$ test image $12,(\mathrm{k})$ test image 13 , (l) test image 14. 


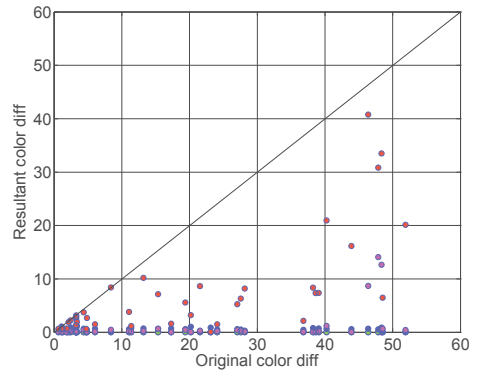

(a)

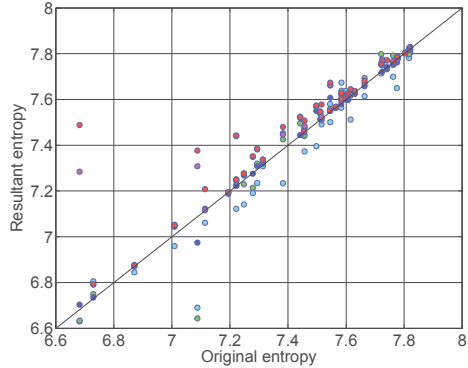

(b)

Figure 16: Scatter plot comparing the results, (a) color difference, (b) information content. Legend: cyan - gray world, green - quadratic, blue - gamma correction, magenta - MOSPO (best color difference criterion), red - MOSPO (best information criterion)

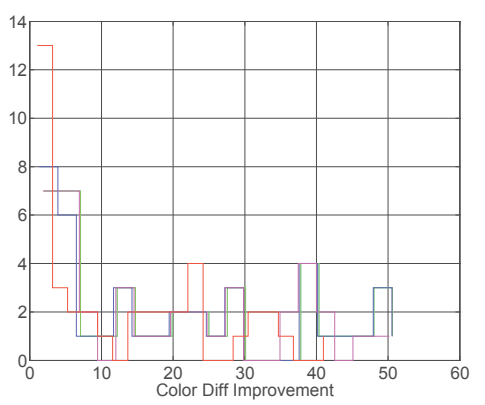

(a)

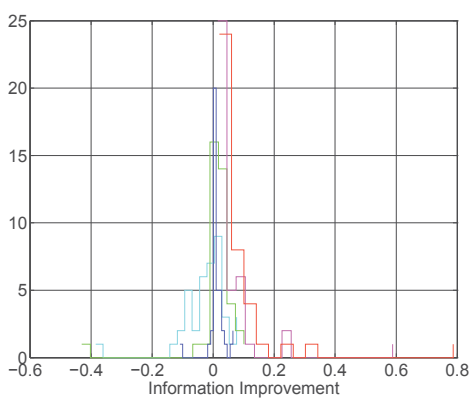

(b)

Figure 17: Histograms comparing the results, (a) color difference, (b) information content. Legend - see Fig. 16.

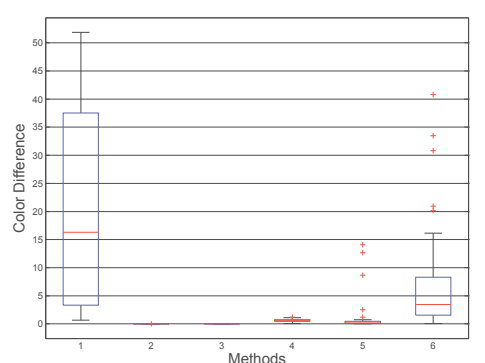

(a)

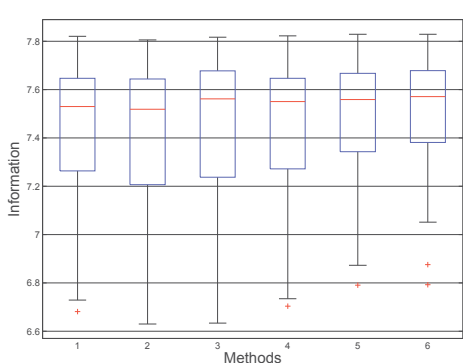

(b)

Figure 18: Box-plots comparing the results, (a) color difference, (b) information content. Column 1: original image, Column 2: gray world correction, Column 3: quadratic correction, Column 4: gamma correction, Column 5: MOSPO with best color difference, Column 6: MOSPO with maximum information gain. 


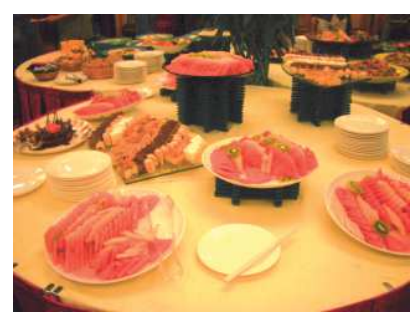

(a)

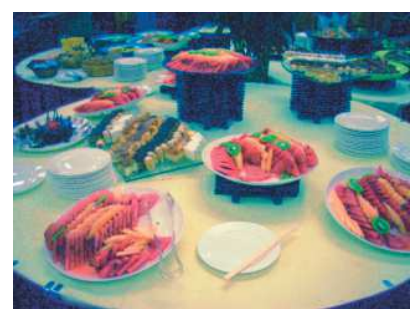

(d)

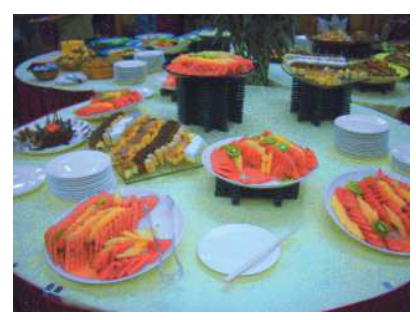

(b)

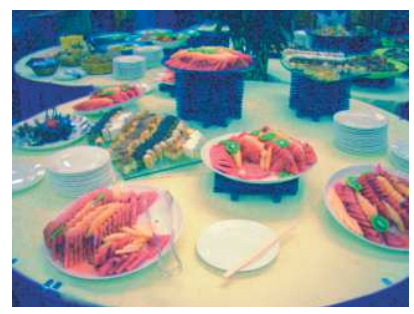

(e)

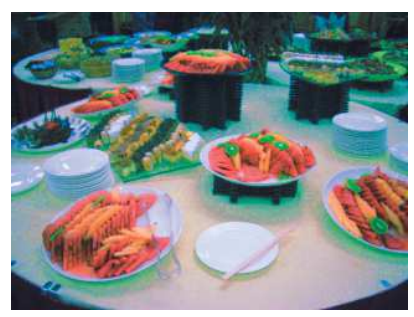

(c)

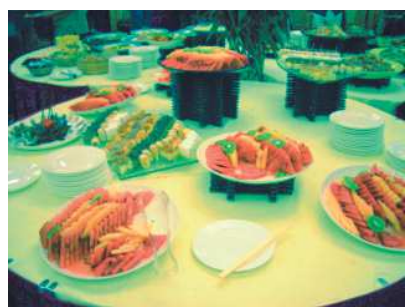

(f)

Figure 19: Image corrected by selected gamma values from Pareto front with best performance in satisfying the gray world assumption, (a) original image, (b) linear correction, (c) quadratic correction, (d) gamma correction, (e) best color difference from MOPSO, (f) best information from MOPSO.

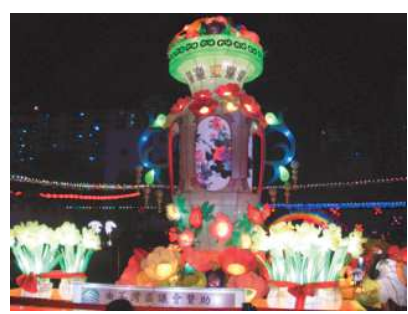

(a)

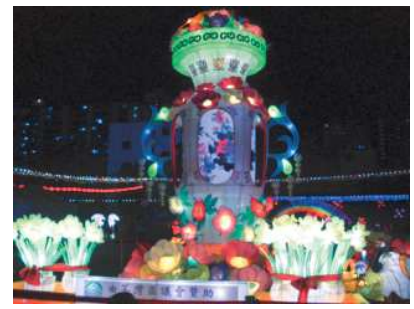

(d)

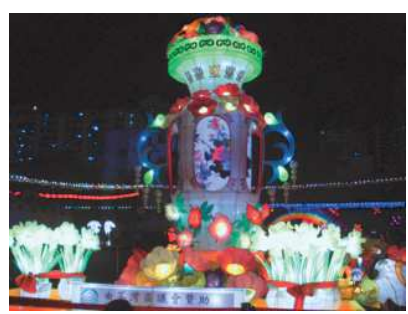

(b)

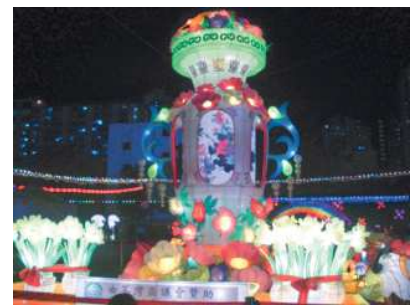

(e)

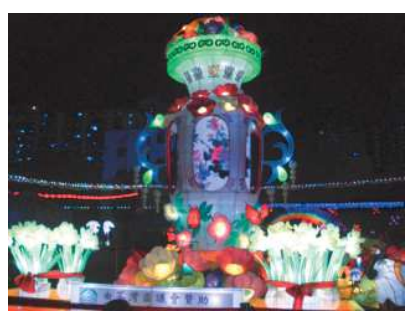

(c)

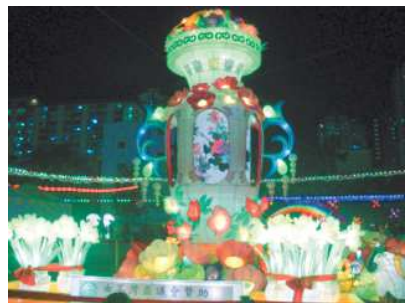

(f)

Figure 20: Image corrected by selected gamma values from Pareto front with best performance in satisfying the information gain criterion, (a) original image, (b) linear correction, (c) quadratic correction, (d) gamma correction, (e) best color difference from MOPSO, (f) best information from MOPSO. 